\title{
REFINED INTERSECTION PRODUCTS AND LIMITING LINEAR SUBSPACES OF HYPERSURFACES
}

\author{
XIAN WU
}

\section{INTRODUCTION}

Let $X$ be a hypersurface of degree $d$ in $\mathbb{P}^{n}$ and $F_{X}$ be the scheme of $\mathbb{P}^{r}$ 's contained in $X$. If $X$ is generic, then $F_{X}$ will have the expected dimension (or empty) and its class is given by the top Chern class of the vector bundle $\operatorname{Sym}^{d} U^{*}$, where $U$ is the universal subbundle on the Grassmannian $G(r+1, n+1)$.

Things become more interesting when $X$ degenerates. For example, when we deform a generic $X$ into a degenerate $X_{0}$, the dimension of $F_{X}$ can jump. In this case, however, there is a subscheme $F_{\text {lim }}$ of $F_{X_{0}}$ which consists of limiting $\mathbb{P}^{r}$ 's in $X_{0}$ with respect to the deformation. For a generic deformation, $F_{\lim }$ will have the expected dimension and its class must also be determined by the top Chern class of Sym ${ }^{d} U^{*}$. This subscheme $F_{\text {lim }}$ of limiting $\mathbb{P}^{r}$ 's will be the main object of study in this paper.

Geometrically, there are two basic questions concerning $F_{\text {lim }}$ that arise naturally. Let us take a simple example. We all know that there are 27 lines in a generic cubic surface. If we degenerate the surface into the union of a plane and a quadric, then there are infinitely many lines in the union. Which 27 lines are the limiting ones and how many of them are in the plane and how many of them are in the quadric? In general, we would like to ask the following questions: How can we identify limiting $\mathbb{P}^{r}$ 's to characterize $F_{\lim }$ ? If $F_{X_{0}}$ has more than one component, what is the local distribution of limiting $\mathbb{P}^{r}$ 's to those components? Such problems in the case of $r=1$ are investigated in [W]. The main tools used there are infinitesimal deformation theory and the theory of Chern classes. In particular, the answer to the example of the cubic surface above is very simple. The intersection of the plane, the quadric, and a cubic (which depends on the deformation) consists of six points. A line in the plane is a limiting line if and only if it passes through any two of those six points. That gives us 15 limiting lines in the plane. On the other hand, a line in the quadric is a limit if and only if it passes through any one of those points. Since for every point in a generic quadric there are two lines that pass through it, we see that there are 12 limiting lines in the quadric.

One of the main goals of this paper is to answer the questions above for arbitrary $r$. In particular, we give a simple but concrete geometric description of $F_{\text {lim }}$ and explicit formulas to compute the local distribution for some generic degenerations. Please see Summary/Theorem 4.1 in Section 4 for the precise statement.

Our formulas (Theorem 3.1 and Proposition 5.3) for the local distribution are computable by using standard techniques in the theory of Chern classes and Schubert calculus. We have included some examples in Section 5. Let us just mention 
one (Example 4) here since it seems to be a bit surprising. We see that there are $321,489 \mathbb{P}^{3}$ 's on a generic cubic hypersurface in $\mathbb{P}^{8}$. When the cubic degenerates into the union of a quadric and a plane, computations from our formulas reveal that all limiting $\mathbb{P}^{3}$ 's are in the plane and none of them is in the quadric! In particular, this implies that $U^{*}$ is not a positive bundle.

Since the total class of $F_{\text {lim }}$ is always equal to the top Chern class of $\mathrm{Sym}^{d} U^{*}$, as a consequence of our main theorems, we get a family of identities involving the Chern classes and the Segre classes of various symmetric powers of $U^{*}$ (Corollary 5.1 and Corollary 5.7). We think that those formulas should hold for any vector bundle of rank $r+1$ over general manifolds. In fact, they should be identities of symmetric polynomials in $r+1$ variables, as we have seen in the case of $r=1$ in $[\mathrm{W}]$. It will be interesting to see if there are other possible applications of such formulas. Those identities can also be found in Section 5 .

The case $r>1$ brings some fundamental changes from $r=1$. The first, there seem to be some technical difficulties to apply the infinitesimal deformation theory in the way used in [W]. However, the main difference is that we can no longer ignore the scheme of $\mathbb{P}^{r}$ 's contained in the singular locus of $X$ if $r>1$. This is a common difficulty that one often faces in doing computations in intersection theory. As a results, we see that the formulas obtained for general $r$ is much more complicated than those for lines and they are not generalizations of the formulas in [W]. On the other hand, we believe that such degeneration problems are not isolated ones and there should be an intrinsic treatment in general. As it turns out, the theory of refined intersection products of Fulton-MacPherson provides a natural way to study our geometric problems. The idea is very simple. Any hypersurface $X$ induces a section $s_{X}$ of $\operatorname{Sym}^{d} U^{*}$. The zero-scheme of $s_{X}$ is nothing but $F_{X}$. Assuming that $F_{X}$ is not empty, we can then consider the fiber square

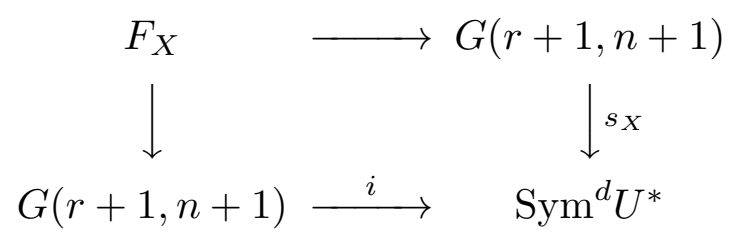

where $i$ is the zero-section imbedding. From this we can construct a class, called $R_{X}$ in this paper, by the refined intersection product of Fulton-MacPherson induced from the above fiber square $[\mathrm{F}$, Chapter 6] [FM]. By well-known facts about refined intersection products and dynamic intersections, this class has the following interesting properties (Theorem 1.3).

(1) It is defined pointwise from data on $X$ and hence is independent of deformations.

(2) Despite (1), its image in the Chow ring of $G(r+1, n+1)$ is always equal to the top Chern class of $\mathrm{Sym}^{d} U^{*}$ and is hence independent of the choice of $X$.

(3) Geometrically, it equals to the class of $F_{X}$ if $X$ is generic and otherwise is equal to the class of the subscheme $F_{\text {lim }}$ of limiting $\mathbb{P}^{r}$ 's with respect to a generic deformation.

(4) Finding the local distribution of limiting $\mathbb{P}^{r}$ 's is equivalent to finding the canonical decomposition of $R_{X}$ in terms of its distinguished varieties.

What makes this formulation more useful is the fact that the above construction and properties are valid for much more general settings under very modest hypotheses. However, unless their distinguished varieties are connected components, which 
is only possible for $r=1$ in the present case, such intersection products and their canonical decompositions are in general difficult to compute. So the construction above does not give us explicit answers directly. Nevertheless, such a formulation sets up the stage for a nice interplay between our geometric problems and intersection theory. In particular, it allows us to apply a powerful theorem of Fulton and Lazarsfeld $[\mathrm{F}][\mathrm{La}]$ on infinitesimal intersection class to conclude that $F_{\text {lim }}$ is determined by the infinitesimal data (Theorem 2.2 and Corollary 2.3). This is one of the key steps in getting our main geometric results. On the other hand, by applying the geometric results back to intersection theory, we are able to find the equivalences of the distinguished varieties and hence the canonical decomposition for refined intersection product $R_{X}$ constructed above (Summary/Theorem 4.1').

While the problem of understanding limiting subschemes arises naturally from studies of algebraic cycles using degeneration techniques, the formulation in the refined intersection product suggests that it may have interesting connections with other problems of a different nature. We have been seeing recently with great excitement the dramatic interplay between string theory and algebraic geometry. In particular, the development has led to some amazing computations [COGP] [M1] [M2] which give striking predictions for the numbers of rational curves in certain Calabi-Yau threefolds and, more recently [GMP], in higher dimensional cases. The Calabi-Yau manifolds in questions are often special types which contain families of rational curves. So one could explain that what those authors have really computed are the numbers of limiting rational curves. Interestingly, no deformations of those Calabi-Yau manifolds are presented in the method (there are only deformations of their "mirrors"). On the other hand, the definition of $R_{X}$ is also purely pointwise and it can be easily generalized to study the limiting rational curves. It is our hope that the basic idea of this paper may lead to more useful results.

This paper is organized as follows. We start our study in Section 1 by giving the construction of $R_{X}$ and interpreting our geometric problems in terms of intersection theory (Theorem 1.3). This is mainly a direct application of some well-known facts about dynamic intersection theory and the refined intersection products of FultonMacPherson.

In Section 2, we study the subscheme $F_{\text {inf }}$ of infinitesimal limiting $\mathbb{P}^{r}$ 's. A main tool here is the infinitesimal intersection class as defined in [F, Chapter 11]. By a theorem of Fulton and Lazarsfeld [F, Chapter 11] [La], this infinitesimal intersection class refines the class of $F_{\text {lim }}$. Moreover, for a generic deformation, $F_{\text {inf }}$ will have the expected dimension and its class is exactly equal to the above infinitesimal intersection class. In other words, limiting $\mathbb{P}^{r}$ 's are determined by the infinitesimal data in that case (Corollary 2.3). With these general facts in place, we proceed with some explicit computations. For this, we consider a generic deformation $\mathcal{D}$ which degenerates $X$ into the union of two hypersurfaces. Up to first order, we may write such a deformation as

$$
\mathcal{D}=\left\{X_{s}=K L+s D\right\}_{s \in \Delta},
$$

where $K, L$, and $D$ are generic hypersurfaces of degree $k, l$, and $d=k+l$, respectively. We will use the same letter to denote a hypersurface and its equation. Let

$$
\sigma_{K}=\left\{\mathbb{P}^{r} \in G(r+1, n+1) \mid \mathbb{P}^{r} \subset K, \mathbb{P}^{r} \cap L \subset D\right\}
$$

and

$$
\sigma_{L}=\left\{\mathbb{P}^{r} \in G(r+1, n+1) \mid \mathbb{P}^{r} \subset L, \mathbb{P}^{r} \cap K \subset D\right\}
$$


The fact (Proposition 2.5) is that $\sigma_{K}$ and $\sigma_{L}$ represent the subschemes of infinitesimal limiting $\mathbb{P}^{r}$ 's and hence limiting $\mathbb{P}^{r}$ 's in $K$ and $L$, respectively. This is done by using the method of deforming ideals adopted from Katz's earlier work [K2].

Turning to Section 3, we will compute the class of $\sigma_{K}$ and the class of $\sigma_{L}$. This has been done in [W] for the case of $r=1$, since the geometric conditions which define $\sigma_{K}$ and $\sigma_{L}$ are the same for $r=1$ and $r>1$. However, the method used in $[\mathrm{W}]$ cannot be generalized here. The main difficulty is that, while $\mathbb{P}^{1}$ 's contained in both $K$ and $L$ can basically be ignored, the same is no longer true for $\mathbb{P}^{r}$ 's if $r$ is greater than 1. In terms of intersection theory, that means while in the case of $r=1$ we can treat the distinguished varieties as though they were connected components, we are now dealing with the excess intersections in a much more complicated way. In fact, a straightforward generalization of the formulas in [W] gives obviously wrong answers. Therefore, some correction terms must be added. Fortunately, nowadays we have the power of the modern technology. After many "experiments" on computers, we were able to guess correct formulas (Theorem 3.1). The actual proof of those formulas follows naturally once we know what to prove.

Section 4 is used primarily to give a summary of the main results obtained in the first three sections for the convenience of readers.

In Section 5, we give some examples and applications of our main results. In particular, as mentioned before, we obtain a family of identities in the characteristic classes of various bundles involving $U^{*}$ (Corollary 5.1 and Corollary 5.7). Those identities seems to be complicated enough to be non-trivial and, on the other hand, to be in a form nice enough to make one suspect that they may have other meanings and applications. Most examples are computed by using a Maple package called "schubert" [KS] written by Katz and Strømme.

Finally, for the convenience of interested readers, we have included schubert code for calculations of two examples in the appendix. We want to thank Katz and Strømme for making this wonderful Maple package available as well as for detailed help on actually using it.

The author is indebted to S. Katz for many valuable discussions. The author also wants to express his thanks to W. Fulton for his encouragement and advice. A part of the work was done while the author was a participant in the NSF Regional Geometry Institute hold at Amherst College during the summer, 1992. The author wants to thank the organizers and the NSF for their generous support.

\section{REFined INTERSECTION PRODUCTS AND LIMITING LINEAR SUBSPACES}

In this section we will lay out the ground work by applying some well-known facts from intersection theory to our geometric problems. Although the statements here are given in forms appropriate for our present setup, most of them can be easily generalized to other settings. The main reference for this section is Fulton's book $[\mathrm{F}]$.

Let $G(r+1, n+1)$ be the Grassmannian of $\mathbb{P}^{r}$ in $\mathbb{P}^{n}$ and $\mathbb{P}^{N}$ be the Hilbert scheme of hypersurfaces of degree $d$ in $\mathbb{P}^{n}$, where

$$
N=\operatorname{dim}\left(\mathbb{P}\left(H^{0}\left(\mathcal{O}_{\mathbb{P}^{n}}(d)\right)\right)\right)=\left(\begin{array}{c}
n+d \\
d
\end{array}\right)-1
$$

Throughout this paper, we will use the same notation for a point of $G(r+1, n+1)$ and its corresponding $\mathbb{P}^{r}$ in $\mathbb{P}^{n}$. Similarly, given an $X$ in $\mathbb{P}^{N}$, we will use same 
$X$ to denote the corresponding hypersurface in $\mathbb{P}^{n}$ and its homogeneous equation. Consider the correspondence

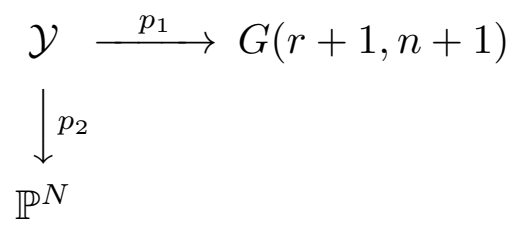

where

$$
\mathcal{Y}=\left\{\left(\mathbb{P}^{r}, X\right) \mid \mathbb{P}^{r} \subset X\right\} \subset G(r+1, n+1) \times \mathbb{P}^{N} .
$$

Given an $X$ in $\mathbb{P}^{N}$, let $F_{X}$ be the scheme of $\mathbb{P}^{r}$ 's in $X$, that is,

$$
F_{X}=p_{1}\left(p_{2}^{-1}(X)\right) .
$$

The fiber over any $\mathbb{P}^{r}$ in $G(r+1, n+1)$ is $\mathbb{P}\left(H^{0}\left(\mathcal{I}_{\mathbb{P}^{r} / \mathbb{P}^{n}}(d)\right)\right)$. From the standard exact sequence

$$
0 \rightarrow \mathcal{I}_{\mathbb{P}^{r} / \mathbb{P}^{n}}(d) \rightarrow \mathcal{O}_{\mathbb{P}^{n}}(d) \rightarrow \mathcal{O}_{\mathbb{P}^{r}}(d) \rightarrow 0,
$$

it is easy to see that $\mathcal{Y}$ is irreducible and smooth of dimension

$$
(r+1)(n-r)+N-\left(\begin{array}{c}
r+d \\
d
\end{array}\right) .
$$

Hence, for a generic $X$ in $\mathbb{P}^{N}, F_{X}$ is either empty or smooth of dimension $m$, where

$$
m=(r+1)(n-r)-\left(\begin{array}{c}
r+d \\
d
\end{array}\right) .
$$

We will assume for a moment that $F_{X}$ is not empty and, in particular, $m$ is nonnegative. For any special $X_{0}$ in $\mathbb{P}^{N}$ such that the dimension of $F_{X_{0}}$ is greater than $m$, we will consider limiting $\mathbb{P}^{r}$ 's in $X_{0}$. The following consideration will be local. For any one-parameter deformation $\mathcal{D}$ of $X_{0}$ in $\mathbb{P}^{N}$, set

$$
\mathcal{Y}_{\mathcal{D}}=\overline{p_{2}^{-1}\left(\mathcal{D}-X_{0}\right)} \text {. }
$$

We define the scheme of limiting $\mathbb{P}^{r}$ 's in $X_{0}$ with respect to $\mathcal{D}$ to be

$$
F_{\text {lim }}^{\mathcal{D}}=\lim _{X \stackrel{\mathcal{D}}{\rightarrow} X_{0}} F_{X}=p_{1}\left(\mathcal{Y}_{\mathcal{D}} \cap p_{2}^{-1}\left(X_{0}\right)\right) .
$$

From the definition, $F_{\text {lim }}^{\mathcal{D}}$ is a subscheme of $F_{X_{0}}$. If $\mathcal{D}$ is generic, then $p_{2}^{-1}(X)$ has dimension $m$ for $X$ in $\mathcal{D}-X_{0}$. Therefore, $F_{\lim }^{\mathcal{D}}$ also has dimension $m$. In this case, we will drop the superscript and denote it by $F_{\text {lim. }}$. Let $U$ be the universal subbundle on $G(r+1, n+1)$ and $\operatorname{Sym}^{d} U^{*}$ be the $d^{\text {th }}$ symmetric power of the dual of $U$. Furthermore, let $c\left(\operatorname{Sym}^{d} U^{*}\right)$ be the total Chern class of $\operatorname{Sym}^{d} U^{*}$ and $s\left(F_{X}, G\right)$ be the Segre class of $F_{X}$ in $G(r+1, n+1)$. 
Theorem 1.3. For any $X$ in $\mathbb{P}^{N}$, there is a well-defined class $R_{X}$ in the Chow $\operatorname{ring} A_{m}\left(F_{X}\right)$ of $F_{X}$ given by

$$
\begin{cases}R_{X}=\left\{c\left(\operatorname{Sym}^{d} U^{*}\right) \cap s\left(F_{X}, G\right)\right\}_{m}, & \text { if } F_{X} \neq \emptyset, \\ R_{X}=0, & \text { if } F_{X}=\emptyset .\end{cases}
$$

such that:

(1) its image in $A_{m}(G(r+1, n+1))$ is independent of $X$ and in fact

$$
j_{*}\left(R_{X}\right)=c_{t o p}\left(\operatorname{Sym}^{d} U^{*}\right) \cap G(r+1, n+1),
$$

where $j$ is the inclusion of $F_{X}$ in $G(r+1, n+1)$.

(2) For a generic $X$ in $\mathbb{P}^{N}, R_{X}$ is equal to the class of $F_{X}$, that is,

$$
R_{X}=\left[F_{X}\right]
$$

(3) For a special $X=X_{0}$ such that $F_{X_{0}}$ has a dimension greater than $m, R_{X_{0}}$ represents the subscheme of limiting $P^{r}$ 's in $X_{0}$ with respect to a generic deformation $\mathcal{D}$. In other words,

$$
R_{X_{0}}=\left[F_{\lim }\right]
$$

(4) If $F_{i}$ is a component of $F_{X_{0}}$, then the part of $R_{X_{0}}$ supported on $F_{i}$ represents the part of limiting $\mathbb{P}^{r}$ 's contained in $F_{i}$. Equivalently,

$$
R_{X_{0}}^{F_{i}}=\left[F_{\lim } \cap F_{i}\right]
$$

Proof of Theorem 1.3. Theorem 1.3 is an immediate consequence of well-known facts from intersection theory once a connection between them is established. We will use constructions and facts from Fulton's book $[\mathrm{F}]$, mainly on refined intersection products and dynamic intersections. Please refer to $[\mathrm{F}]$ for further details.

For any $X$ in $P^{N}, X$ induces a section $s_{X}$ of $\operatorname{Sym}^{d} U^{*}$. In fact, $\operatorname{Sym}^{d} U^{*}$ is generated by such sections. This follows from the fact that the natural map

$$
H^{0}\left(\mathcal{O}_{\mathbb{P}^{n}}(d)\right)_{G(r+1, n+1)} \rightarrow \mathrm{Sym}^{d} U^{*}
$$

is surjective. The zero-scheme $Z\left(s_{X}\right)$ of $s_{X}$ consists of those $\mathbb{P}^{r}$ 's which are contained in $X$. In other words, $Z\left(s_{X}\right)$ is equal to $F_{X}$. If $F_{X}$ is empty, then using the splitting principle it is easy to see that the top Chern class of $\operatorname{Sym}^{d} U^{*}$ is zero. We are therefore done in this case. On the other hand, if $F_{X}$ is not empty, we can consider the fiber square

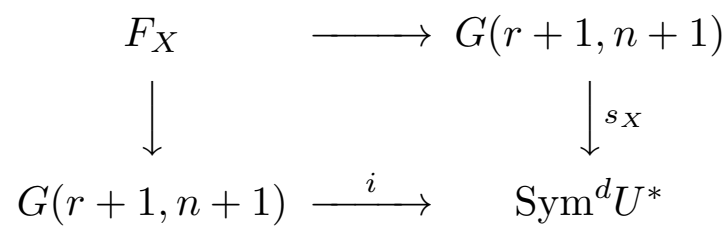

where $i$ is the zero-section imbedding. We define $R_{X}$ to be the refined intersection product induced from fiber square (1.5) as given in [F, Chapter 6]. In other words,

$$
R_{X}=i^{!}([G(r+1, n+1)])=i^{*}\left(C_{F_{X}}\right),
$$


where $C_{F_{X}}$ is the normal cone to $F_{X}$ in $G(r+1, n+1), i^{!}$is the refined Gysin homomorphism induced from (1.5), and $i^{*}$ is the Gysin homomorphism induced from $i$. Since the normal bundle to $G(r+1, n+1)$ in $\operatorname{Sym}^{d} U^{*}$ is $\operatorname{Sym}^{d} U^{*}$ itself, $R_{X}$ therefore is a well-defined class in $A_{m}\left(F_{X}\right)$ given by

$$
R_{X}=\left\{c\left(\operatorname{Sym}^{d} U^{*}\right) \cap s\left(F_{X}, G\right)\right\}_{m}
$$

and its image in $A_{*}(G(r+1, n+1))$ is equal to

$$
c_{\text {top }}\left(\operatorname{Sym}^{d} U^{*}\right) \cap G(r+1, n+1) .
$$

To see claim (2), recall that $\mathrm{Sym}^{d} U^{*}$ is generated by sections induced from hypersurfaces of degree $d$ in $\mathbb{P}^{n}$. Therefore, for a generic $X$ in $\mathbb{P}^{N}$, the induced section $s_{X}$ is transverse to the zero-section. Hence, by the definition, $R_{X}$ is equal to the class of $F_{X}$. (This also gives another way to see that $F_{X}$ is smooth of dimension $m$.) Claim (3) and Claim (4) will now follow from the definition of $F_{\lim }$ and general facts about dynamic intersections. Taking a deformation $\mathcal{D}$ and deforming fiber square (1.5) accordingly, we get the fiber square

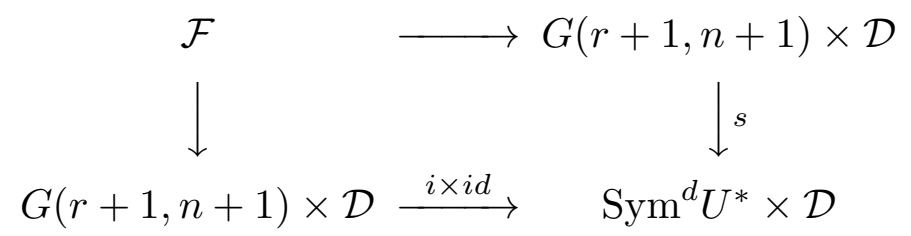

where

$$
s(*, X)=\left(s_{X}(*), X\right) .
$$

Define $\mathcal{R}$ to be the refined intersection product in $A_{m+1}(\mathcal{F})$ induced from fiber square (1.6). Notice that $\mathcal{F}$ is a scheme over $D$ with the fiber over $X$ being $F_{X}$. Since the rational equivalence is preserved when passing from a family to its fibers, $\mathcal{R}$ defines a family of classes over $\mathcal{D}$ with the fiber over $X$ being $\mathcal{R}_{X}$. Next, let us recall the definition of the limit of $\mathcal{R}_{X}$. Assume

$$
\mathcal{R}=\sum_{i} n_{i}\left[\mathcal{V}_{i}\right]
$$

where $\mathcal{V}_{i}$ are subvarieties of $\mathcal{F}$. Let

$$
\mathcal{V}_{i}^{*}=\mathcal{V}_{i}-F_{X_{0}}
$$

We define the limit of $\mathcal{R}_{X}$ to be

$$
\lim _{X \stackrel{\mathcal{D}}{\rightarrow} X_{0}} \mathcal{R}_{X}=\sum_{i} n_{i}\left[\overline{\mathcal{V}_{i}^{*}} \cap F_{X_{0}}\right]
$$

It is easy to check that this limit is a well-defined class. An important fact in dynamic intersections [F, Chapter 11] is that

$$
\lim _{X \stackrel{\mathcal{D}}{\rightarrow} X_{0}} \mathcal{R}_{X}=\mathcal{R}_{X_{0}} .
$$


On the other hand, fiber square (1.6) can be considered as a family of fiber squares over $\mathcal{D}$ with fibers over $X$ being fiber square (1.5). From this, we see that $\mathcal{R}_{X}$ is nothing but $R_{X}$. Therefore, for a generic deformation $\mathcal{D}$,

$$
\begin{aligned}
R_{X_{0}}= & \mathcal{R}_{X_{0}} \\
= & \lim _{X \mathcal{D} \rightarrow X_{0}} \mathcal{R}_{X} \\
= & \lim _{X \mathcal{D}} R_{X} R_{X} \\
= & \lim _{X \mathcal{D}}\left[F_{X}\right] \\
= & {\left[\lim _{X \mathcal{D}} F_{X} X_{X}\right] } \\
= & {\left[F_{\lim }\right] . }
\end{aligned}
$$

From the construction, it is clear that Claim (4) also follows.

Since $F_{X}$ gives a positive class if it is not empty, we have the following corollary.

Corollary 1.7. For a generic $X$, the scheme $F_{X}$ is empty if and only if $c_{t o p}\left(\operatorname{Sym}^{d} U^{*}\right)$ is zero.

Remark. The scheme $F_{X}$ can be empty even if $m$ is non-negative. For example, it is well-known [GH, Chapter 6] that a smooth quadric in $\mathbb{P}^{n}$ contains no $\mathbb{P}^{r}$ if $r$ is strictly greater than $(n-1) / 2$. On the other hand, for $d=2$ in $(1.2), m$ is non-negative if $r$ is less than or equal to $2(n-1) / 3$.

From Theorem 1.3, it is clear now that the next step is to understand $R_{X}$. Of course, the image of $R_{X}$ in $A_{m}(r+1, n+1)$ is always equal to the top Chern class of $\operatorname{Sym}^{d} U^{*}$. Our geometric goal is to identify $F_{\text {lim }}$ in $F_{X}$ if the dimension of $F_{X}$ is greater than $m$. In terms of $R_{X}$, that means to compute its canonical decomposition. For example, $F_{X}$ will have $l$ components $F_{X_{i}}, 1 \leq i \leq l$, if we degenerate a generic hypersurface into a normal crossing of $l$ generic hypersurfaces

$$
X=\bigcup_{i=1}^{l} X_{i}, \quad \sum_{i=1}^{l} \operatorname{deg} X_{i}=d .
$$

Those $F_{X_{i}}$ are all distinguished varieties of $R_{X}$ if we assume that they are all irreducible. (This may not be the case in general, but that is not important for our discussion.) To find limiting $\mathbb{P}^{r}$ 's in each $X_{i}$ is equivalent to find the equivalence, or the contribution, of corresponding distinguished variety to $R_{X}$. Unfortunately, unless those varieties are also connected components, such a decomposition is in general difficult to compute. In fact, one cannot expect an explicit formula in such a general setting. The following is how we will proceed. First, we will see that the problem can be reduced to a problem of the infinitesimal nature. To make more explicit computations, we then turn our attention to degenerations such that $X_{0}$ has two components and identify infinitesimal limiting $\mathbb{P}^{r}$ 's in each component geometrically. Finally, the class of limiting $\mathbb{P}^{r}$ 's in each component will be computed from those geometric conditions. Those steps will be carried out in next two sections. 


\section{INFINITESIMAL INTERSECTION CLASSES AND \\ INFINITESIMAL LIMITING LINEAR SUBSPACES}

In this section we will study the subscheme of infinitesimal limiting $\mathbb{P}^{r}$ 's. Our considerations therefore will be all local. We will use a theorem of Fulton and Lazarsfeld on infinitesimal intersection classes. This makes it possible to study the subscheme of limiting $\mathbb{P}^{r}$ 's from infinitesimal data. The theorem of Fulton and Lazarsfeld implies that this principle is true in much more general settings. Please refer to [F, Chapter 11] for further details.

Let us first recall a construction of [F, Chapter 11]. For a given $X_{0}$ in $\mathbb{P}^{N}$ and a one-parameter deformation $\mathcal{D}$ of $X_{0}$, we consider the imbedding $s$ as given in (1.6)

$$
G(r+1, n+1) \times \mathcal{D} \stackrel{s}{\longrightarrow} \operatorname{Sym}^{d} U^{*} \times \mathcal{D} .
$$

Let $T$ be the tangent space to $\mathcal{D}$ at $X_{0}$ and $T_{G}$ be the trivial line bundle $T \times G(r+$ $1, n+1)$ on $G(r+1, n+1)$. Let $N_{X_{0}}$ be the normal bundle of

$$
s_{X_{0}}(G(r+1, n+1)) \simeq s\left(G(r+1, n+1), X_{0}\right)
$$

in $\operatorname{Sym}^{d} U^{*}$. Since $F_{X_{0}}$ is the zero-scheme of $s_{X_{0}}$, we have that

$$
\left.N_{X_{0}}\right|_{F_{X_{0}}}=\left.\operatorname{Sym}^{d} U^{*}\right|_{F_{X_{0}}}
$$

and we will call this restriction $N$. From (2.1), a basis $\partial / \partial t$ of $T$ will induce a section of $T_{G}$ and hence a section of $N_{X_{0}}$ by the Kodaira-Spencer homomorphism. Let $s_{T}$ be the restriction of the above section of $N_{X_{0}}$ on $N$. Hence $s_{T}$ is a section of $\operatorname{Sym}^{d} U^{*}$ over $F_{X_{0}}$. Let $C$ be the normal cone to $F_{X_{0}}$ in $G(r+1, n+1)$. Notice that $C$ is a purely $(n-r)(r+1)$-dimensional subscheme of $N$. Let

$$
F_{\text {inf }}=s_{T}^{-1}(C)
$$

be the subscheme of infinitesimal limiting $\mathbb{P}^{r}$ 's in $F_{X_{0}}$. The infinitesimal intersection class $R_{\text {inf }}$ is then defined to be

$$
R_{\text {inf }}=s_{T}^{!}(C) \in A_{m}\left(F_{\text {inf }}\right) .
$$

We can now state a theorem of Fulton and Lazarsfeld [F] [La].

Theorem 2.2 (Fulton and Lazarsfeld). We have the following inclusions

$$
F_{\text {lim }} \hookrightarrow F_{\text {inf }} \stackrel{j}{\hookrightarrow} F_{X_{0}} .
$$

Moreover, $R_{\mathrm{inf}}$ refines $R_{X_{0}}$, that is,

$$
j_{*}\left(R_{\mathrm{inf}}\right)=R_{X_{0}} .
$$

Proof. Since $s_{X}$ is a regular imbedding of $G(r+1, n+1)$ in $\operatorname{Sym}^{d} U^{*}$, the refined intersection product $\mathcal{R}$ defined in the proof of Theorem 1.3 can also be defined from the transform of fiber square (1.6)

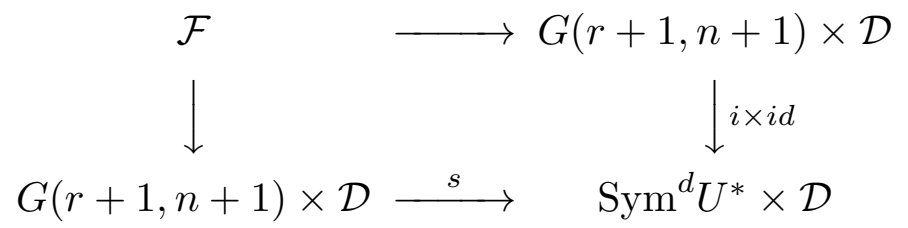

Similarly, consider $R_{X}$ as the refined intersection product induced from the transform of fiber square (1.5). Now apply Theorem 11.2 of $[\mathrm{F}]$.

If we take $\mathcal{D}$ to be general enough, $F_{\text {inf }}$ will have the expected dimension and $R_{\text {inf }}$ then represents the class of $F_{\text {inf. }}$. 
Corollary 2.3. For a generic deformation $\mathcal{D}$,

$$
\left[F_{\text {lim }}\right]=\left[F_{\text {inf }}\right]=R_{X_{0}}
$$

and the subscheme $F_{\lim }$ of limiting $\mathbb{P}^{r}$ 's is hence determined by the infinitesimal data.

We will now make some explicit computations about $F_{\text {inf. }}$ For this, we will restrict our attention to a simple type of degenerations. To be more precise, we will consider

$$
\mathcal{D}=\left\{X_{s}\right\}_{s \in \Delta}
$$

a semistable degeneration of generic hypersurfaces of degree $d$ in $\mathbb{P}^{n}$ such that $X_{0}$ has two irreducible components. As indicated in the introduction, we will use the same letter to denote a hypersurface and its equation. Up to first order, we may write that

$$
\mathcal{D}=\left\{X_{s}=K L+s D\right\}_{s \in \Delta},
$$

where $K, L$, and $D$ are generic hypersurfaces of degree $k, l$, and $d=k+l$, respectively. We define

$$
\sigma_{K}=\left\{\mathbb{P}^{r} \in G(r+1, n+1) \mid \mathbb{P}^{r} \subset K, \mathbb{P}^{r} \cap L \subset D\right\}
$$

and

$$
\sigma_{L}=\left\{\mathbb{P}^{r} \in G(r+1, n+1) \mid \mathbb{P}^{r} \subset L, \mathbb{P}^{r} \cap K \subset D\right\} .
$$

Notice that $\sigma_{K} \cup \sigma_{L}$ is a subset of $F_{X_{0}}$ subject to the given geometric conditions. The extra conditions are imposed exactly by the first order obstructions to the deformation. To be more precise, we have the following proposition.

Proposition 2.5. For a generic degeneration (2.4),

$$
F_{\text {inf }}=\sigma_{K} \cup \sigma_{L}
$$

Proof of Proposition 2.5. We will use the method of deforming ideals to study the first order obstruction on $\mathbb{P}^{r}$ 's in $F_{X_{0}}$ as Katz [K2] did in the case of quintic threefolds. For any $\mathbb{P}^{r}$ in $F_{X_{0}}$, we have that either $\mathbb{P}^{r}$ is contained in $K$ or $\mathbb{P}^{r}$ is contained in $L$. Assume that $\mathbb{P}^{r}$ is contained in $K$ and it deforms with $X_{s}$. Take a local trivialization of $\mathcal{D}$ and a family

$$
\mathbb{P}_{s}^{r} \subset X_{s}, \quad \mathbb{P}_{0}^{r}=\mathbb{P}^{r} .
$$

Let $I_{s}$ be the ideal of $\mathbb{P}_{s}^{r}$ in $\mathbb{P}^{n}$ and

$$
I_{s}=\left\{f_{1}(s), f_{2}(s), \cdots, f_{t}(s)\right\} .
$$

Since $X_{s}$ is in $I_{s}$, we have that

$$
X_{s}=\sum_{i=1}^{t} a_{i}(s) f_{i}(s)=K L+s D+s^{2} E+\cdots .
$$


Therefore, we have infinitesimally that

$$
\left\{\begin{aligned}
K L & =\sum_{i=1}^{t} a_{i}(0) f_{i}(0) \\
D & =\sum_{i=1}^{t} a_{i}(0) f_{i}^{\prime}(0)+\sum_{i=1}^{t} a_{i}^{\prime}(0) f_{i}(0)
\end{aligned}\right.
$$

Since $\mathbb{P}^{r}$ is in $K$,

$$
K=\sum_{i=1}^{t} b_{i} f_{i}(0)
$$

Therefore, from the first equation in (2.6), we may choose $K$ and $L$ such that

$$
a_{i}(0)=b_{i} L .
$$

Substituting this into the second equation in (2.6), we get

$$
D=L \sum_{i=1}^{t} b_{i} f_{i}^{\prime}(0)+\sum_{i=1}^{t} a_{i}^{\prime}(0) f_{i}(0) \text {. }
$$

Since

$$
\left.f_{i}(0)\right|_{\mathbb{P}^{r}}=0
$$

we then have that

$$
\left.D\right|_{\mathbb{P}^{r}}=\left.\left.L\right|_{\mathbb{P}^{r}}\left(\sum_{i=1}^{t} b_{i} f_{i}^{\prime}(0)\right)\right|_{\mathbb{P}^{r}} .
$$

The equation above implies that

$$
\left.D\right|_{\mathbb{P}^{r}}(p)=0, \quad \text { whatever }\left.L\right|_{\mathbb{P}^{r}}(p)=0 .
$$

This is just the definition of $\sigma_{K}$.

Our next step will be to compute the class of $\sigma_{K}$ and the class of $\sigma_{L}$ from the given geometric conditions. For this, we will turn to Section 3.

\section{Formulas FOR $\sigma_{K}$ AND $\sigma_{L}$}

Our goal in this section is to derive formulas for the classes of $\sigma_{K}$ and $\sigma_{L}$ in the Chow ring $A_{*}(G(r+1, n+1))$ from their definitions. That together with the results in Section 1 and Section 2 will enable us to compute the local distribution of limiting $\mathbb{P}^{r}$ 's and hence the canonical decomposition of $R_{X_{0}}$. To simplify the notation, we will identify an element in $A^{*}(G(r+1, n+1))$ with its dual in $A_{*}(G(r+1, n+1))$.

Retaining the notation in previous sections, we consider first the projective bundle

$$
\mathbb{P}\left(\operatorname{Sym}^{l} U^{*}\right) \stackrel{\pi_{l}}{\longrightarrow} G(r+1, n+1) .
$$

Let

$$
T_{l}=\mathcal{O}(-1)
$$

be the tautological subbundle on $\mathbb{P}\left(\operatorname{Sym}^{l} U^{*}\right)$. Similarly, let $T_{k}$ be the tautological subbundle on the projective bundle

$$
\mathbb{P}\left(\operatorname{Sym}^{k} U^{*}\right) \stackrel{\pi_{k}}{\longrightarrow} G(r+1, n+1) .
$$

For a given vector bundle $E$, we will use $c_{t o p}(E)$ to denote the top Chern class of $E$. Recall also that we have $d=k+l$. 
Theorem 3.1. Let $\left[\sigma_{K}\right]$ be the class of $\sigma_{K}$ in $A_{*}(G(r+1, n+1))$. We have that $(3.2)$

$$
\left[\sigma_{K}\right]=c_{\text {top }}\left(\operatorname{Sym}^{k} U^{*}\right) \pi_{l *}\left(c_{t o p}\left(\pi_{l}^{*} \operatorname{Sym}^{d} U^{*} /\left(\pi_{l}^{*} \operatorname{Sym}^{k} U^{*} \otimes T_{l}\right)\right) c_{t o p}\left(\pi_{l}^{*} \operatorname{Sym}^{l} U^{*} / T_{l}\right)\right) .
$$

The corresponding formula holds for the class of $\sigma_{L}$, that is,

$$
\left[\sigma_{L}\right]=c_{\text {top }}\left(\operatorname{Sym}^{l} U^{*}\right) \pi_{k *}\left(c_{t o p}\left(\pi_{k}^{*} \operatorname{Sym}^{d} U^{*} /\left(\pi_{k}^{*} \operatorname{Sym}^{l} U^{*} \otimes T_{k}\right)\right) c_{t o p}\left(\pi_{k}^{*} \operatorname{Sym}^{k} U^{*} / T_{k}\right)\right) .
$$

Proof of Theorem 3.1. Recall that

$$
\begin{aligned}
\sigma_{K} & =\left\{\mathbb{P}^{r} \in G(r+1, n+1) \mid \mathbb{P}^{r} \subset K, \mathbb{P}^{r} \cap L \subset D\right\} \\
& =\left\{\mathbb{P}^{r} \in G(r+1, n+1) \mid \mathbb{P}^{r} \subset K\right\} \cap\left\{\mathbb{P}^{r} \in G(r+1, n+1) \mid \mathbb{P}^{r} \cap L \subset D\right\} .
\end{aligned}
$$

Since $K$ is a generic hypersurface of degree $k$,

$$
\left[\sigma_{K}\right]=c_{t o p}\left(\operatorname{Sym}^{k} U^{*}\right)[B(D, L)],
$$

where $[B(D, L)]$ is the class of

$$
B(D, L)=\left\{\mathbb{P}^{r} \in G(r+1, n+1) \mid \mathbb{P}^{r} \cap L \subset D\right\} .
$$

To see $[B(D, L)]$, we will first rewrite $\mathbb{P}\left(\operatorname{Sym}^{l} U^{*}\right)$ as

$$
\mathbb{P}\left(\operatorname{Sym}^{l} U^{*}\right)=\left\{\left(X_{l}, \mathbb{P}^{r}\right) \mid X_{l} \subset \mathbb{P}^{r}\right\} .
$$

Notice that, for $l>1, \mathbb{P}^{r}$ in $\left(X_{l}, \mathbb{P}^{r}\right)$ is uniquely determined by $X_{l}$ and we will call this $\mathbb{P}^{r}$ the supporting plane of $X_{l}$. Therefore, geometrically, we can identify $\mathbb{P}\left(\operatorname{Sym}^{l} U^{*}\right)$ as the space of degree $l$ subvarieties $X_{l}$ of dimension $(r-1)$ in $\mathbb{P}^{n}$ such that $X_{l}$ is contained in some $\mathbb{P}^{r}$. In the case of $l=1, \mathbb{P}\left(\mathrm{Sym}^{l} U^{*}\right)$ can be identified as the space of marked $\mathbb{P}^{r-1}$ 's in $\mathbb{P}^{n}$ with the mark to be a $\mathbb{P}^{r}$ assigned to each $\mathbb{P}^{r-1}$. Such a $\mathbb{P}^{r}$ is taken from all $\mathbb{P}^{r}$ 's containing the given $X_{1}=\mathbb{P}^{r-1}$ and we will also call it the supporting plane of $X_{1}$. With such an understanding, we will from now on denote an element of $\mathbb{P}\left(\mathrm{Sym}^{l} U^{*}\right)$ just by $X_{l}$. Now, consider the commutative diagram

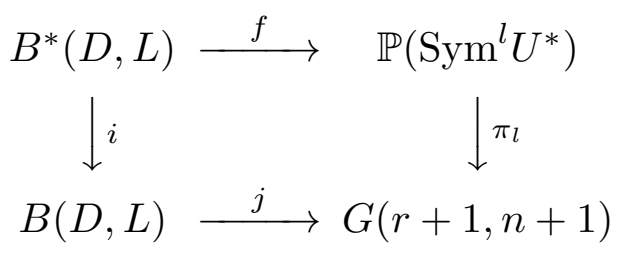

where

$$
B^{*}(D, L)=B(D, L)-\left\{\mathbb{P}^{r} \mid \mathbb{P}^{r} \subset L\right\},
$$

$i$ and $j$ are inclusions, and $f$ is defined by

$$
f\left(\mathbb{P}^{r}\right)=\mathbb{P}^{r} \cap L .
$$

From the definition, we see that $f$ is 1-to-1. Moreover, $f$ can be considered as a rational map on $B(D, L)$. Since

$$
\left.\pi_{l} \circ f\right|_{B^{*}(D, L)}=i d,
$$


we therefore have that

$$
\begin{aligned}
{[B(D, L)] } & =\left[\overline{B^{*}(D, L)}\right] \\
& =\left[\overline{\pi_{l} \circ f\left(B^{*}(D, L)\right)}\right] \\
& =\left[\pi_{l}\left(\overline{f\left(B^{*}(D, L)\right)}\right)\right] \\
& =\pi_{l *}\left(\left[\overline{f\left(B^{*}(D, L)\right)}\right]\right) .
\end{aligned}
$$

The following lemma is needed to complete our argument and its proof will be given later.

\section{Lemma 3.5.}

$$
\overline{f\left(B^{*}(D, L)\right)}=\left\{X_{l} \mid X_{l} \subset D\right\} \cap\left\{X_{l} \mid X_{l} \subset L\right\} .
$$

To see how to use Lemma 3.5 to derive formula (3.2), consider the subbundle

$$
\pi_{l}^{*} \operatorname{Sym}^{k} U^{*} \otimes T_{l} \hookrightarrow \pi_{l}^{*} \operatorname{Sym}^{d} U^{*}
$$

on $\mathbb{P}\left(\operatorname{Sym}^{l} U^{*}\right)$. (Recall that $d=k+l$.) This defines a quotient bundle $Q_{d}$ on $\mathbb{P}\left(\mathrm{Sym}^{l} U^{*}\right)$ which fits into the exact sequence

$$
0 \rightarrow \pi_{l}^{*} \operatorname{Sym}^{k} U^{*} \otimes T_{l} \rightarrow \pi_{l}^{*} \operatorname{Sym}^{d} U^{*} \rightarrow Q_{d} \rightarrow 0
$$

Hypersurface $D$ induces a section of $\operatorname{Sym}^{d} U^{*}$ and hence a section $s_{D}$ of $Q_{d}$. The zero-scheme of $s_{D}$ consists exactly of $X_{l}$ 's which are contained in $D$. In fact, at each $X_{l}$ in $\mathbb{P}\left(\operatorname{Sym}^{l} U^{*}\right)$, the value of the section induced by $D$ in $\pi_{l}^{*} \operatorname{Sym}^{d} U^{*}$ is given by the intersection of $D$ with the supporting plane of $X_{l}$. It lies in $\pi_{l}^{*} \operatorname{Sym}^{k} U^{*} \otimes T_{l}$ if and only if this intersection is the union of $X_{l}$ and some degree $k$ hypersurface in the supporting plane. On the other hand, it is easy to see that $Q_{d}$ is generated by sections induced from hypersurfaces of degree $d$ in $\mathbb{P}^{n}$. Since $D$ is generic, $s_{D}$ is therefore a regular section of $Q_{d}$. Hence, by the localized top Chern class theorem,

$$
\left[\left\{X_{l} \mid X_{l} \subset D\right\}\right]=\left[Z\left(s_{D}\right)\right]=c_{\text {top }}\left(Q_{d}\right)=c_{\text {top }}\left(\pi_{l}^{*} \operatorname{Sym}^{d} U^{*} /\left(\pi_{l}^{*} \operatorname{Sym}^{k} U^{*} \otimes T_{l}\right)\right) .
$$

In a similar way, we have that

$$
\left[\left\{X_{l} \mid X_{l} \subset L\right\}\right]=c_{t o p}\left(\pi_{l}^{*} \operatorname{Sym}^{l} U^{*} / T_{l}\right)
$$

Lemma 3.5 and formula (3.4) together now yield the formula for $[B(D, L)]$ and hence formula $(3.2)$ for $\left[\sigma_{K}\right]$, that is,

$$
\begin{aligned}
{\left[\sigma_{K}\right] } & =c_{t o p}\left(\operatorname{Sym}^{k} U^{*}\right)[B(D, L)] \\
& =c_{t o p}\left(\operatorname{Sym}^{k} U^{*}\right) \pi_{l *}\left(c_{t o p}\left(\pi_{l}^{*} \operatorname{Sym}^{d} U^{*} /\left(\pi_{l}^{*} \operatorname{Sym}^{k} U^{*} \otimes T_{l}\right)\right) c_{t o p}\left(\pi_{l}^{*} \operatorname{Sym}^{l} U^{*} / T_{l}\right)\right) .
\end{aligned}
$$

To complete our proof, we still need to show Lemma 3.5 that

$$
\overline{f\left(B^{*}(D, L)\right)}=\left\{X_{l} \mid X_{l} \subset D\right\} \cap\left\{X_{l} \mid X_{l} \subset L\right\}
$$


To simplify the notation, we will call the right-hand side of (3.6) B. Recall that

$$
Q_{d}=\pi_{l}^{*} \operatorname{Sym}^{k+l} U^{*} /\left(\pi_{l}^{*} \operatorname{Sym}^{k} U^{*} \otimes T_{l}\right) .
$$

Let also

$$
Q_{l}=\pi_{l}^{*} \operatorname{Sym}^{l} U^{*} / T_{l} .
$$

As we have shown, $B$ is the intersection of the zero-scheme of $s_{D}$ and the zeroscheme of $s_{L}$, where $s_{D}$ and $s_{L}$ are sections of $Q_{d}$ and $Q_{l}$ induced by $D$ and $L$, respectively. Moreover, those two quotient bundles $Q_{d}$ and $Q_{l}$ are generated by sections induced by hypersurfaces of degree $d$ and degree $l$ in $\mathbb{P}^{n}$, respectively. Since $D$ and $L$ are generic, we see that $B$ is a smooth subvariety of pure dimension in $\mathbb{P}\left(\operatorname{Sym}^{l} U^{*}\right)$. Let

$$
B^{*}=\left\{X_{l} \in B \mid \text { the supporting plane of } X_{l} \text { is not contained in } L\right\} .
$$

It is easy to see from the definition of $f$ that

$$
f\left(B^{*}(D, L)\right)=B^{*} .
$$

Therefore, to show (3.6), it is enough to show that $B-B^{*}$ is proper in $B$. To see this, notice that

$$
\begin{aligned}
B-B^{*} & =\left\{X_{l} \mid X_{l} \subset D\right\} \cap\left\{X_{l} \mid X_{l} \subset \mathbb{P}^{r} \subset L\right\} \\
& =\left\{X_{l} \mid X_{l} \subset D\right\} \cap\left(\bigcup_{\mathbb{P}^{r} \subset L} \pi_{l}^{-1}\left(\mathbb{P}^{r}\right)\right) .
\end{aligned}
$$

Since $D$ is generic, it is enough for us to show that

$$
\operatorname{dim}\left(\bigcup_{\mathbb{P}^{r} \subset L} \pi_{l}^{-1}\left(\mathbb{P}^{r}\right)\right)<\operatorname{dim}\left(\left\{X_{l} \mid X_{l} \subset L\right\}\right) .
$$

This is easy to see. In fact, we have either

$$
\bigcup_{\mathbb{P}^{r} \subset L} \pi^{-1}\left(\mathbb{P}^{r}\right)=\emptyset
$$

or

$$
\begin{aligned}
\operatorname{dim}\left(\bigcup_{\mathbb{P}^{r} \subset L} \pi^{-1}\left(\mathbb{P}^{r}\right)\right) & =\operatorname{rank}\left(\operatorname{Sym}^{l} U^{*}\right)-1+\left(\operatorname{dim}(G(r+1, n+1))-\operatorname{rank}\left(\operatorname{Sym}^{l} U^{*}\right)\right) \\
& =\operatorname{dim}(G(r+1, n+1))-1 .
\end{aligned}
$$

On the other hand,

$$
\begin{aligned}
\operatorname{dim}\left(\left\{X_{l} \mid X_{l} \subset L\right\}\right) & =\operatorname{dim}\left(\mathbb{P}\left(\operatorname{Sym}^{l} U^{*}\right)\right)-\operatorname{rank}\left(Q_{l}\right) \\
& =\operatorname{dim}(G(r+1, n+1))+\operatorname{rank}\left(\operatorname{Sym}^{l} U^{*}\right)-1-\left(\operatorname{rank}\left(\operatorname{Sym}^{l} U^{*}\right)-1\right) \\
& =\operatorname{dim}(G(r+1, n+1)) .
\end{aligned}
$$

Therefore, $B-B^{*}$ is either empty or a divisor in $B$. This completes our proof of Lemma 3.5 and hence our proof of formula (3.2). By interchanging $l$ and $k$, the same argument will yield formula (3.3) for the class of $\left[\sigma_{L}\right]$.

Theorem 3.1 gives us a tool to compute the classes of $\left[\sigma_{K}\right]$ and $\left[\sigma_{L}\right]$ using standard techniques in intersection theory. We have made some computations using a Maple package written by Katz and Strømme. Computations of examples have played important roles in discovering Theorem 3.1. In fact, many of them were made before Theorem 3.1 was proved. A part of those examples along with other things will be given in Section 5 . 


\section{Summary of main Results obtained so far}

We will summarize the main results obtained so far in this section. For more details on notation, please refer to corresponding theorems and formulas as indicated.

Summary/Theorem 4.1. For a generic degeneration

$$
\mathcal{D}=\left\{X_{s}=K L+s D+\cdots\right\}_{s \in \Delta},
$$

there are no higher order obstructions to deform $\mathbb{P}^{r}$ with $X_{0}$. The subscheme $F_{\lim }$ of limiting $\mathbb{P}^{r}$ 's is hence determined by the infinitesimal data. In particular,

$$
F_{\lim }=\sigma_{K} \cup \sigma_{L}
$$

where, as in (2.5),

$$
\sigma_{K}=\left\{\mathbb{P}^{r} \in G(r+1, n+1) \mid \mathbb{P}^{r} \subset K, \mathbb{P}^{r} \cap L \subset D\right\}
$$

and

$$
\sigma_{L}=\left\{\mathbb{P}^{r} \in G(r+1, n+1) \mid \mathbb{P}^{r} \subset L, \mathbb{P}^{r} \cap K \subset D\right\}
$$

consist of limiting $\mathbb{P}^{r}$ 's in $K$ and $L$, respectively. Furthermore, their classes can be computed by formulas (3.2) and (3.3)

$$
\left[\sigma_{K}\right]=c_{\text {top }}\left(\operatorname{Sym}^{k} U^{*}\right) \pi_{l *}\left(c_{\text {top }}\left(\pi_{l}^{*} \operatorname{Sym}^{d} U^{*} /\left(\pi_{l}^{*} \operatorname{Sym}^{k} U^{*} \otimes T_{l}\right)\right) c_{t o p}\left(\pi_{l}^{*} \operatorname{Sym}^{l} U^{*} / T_{l}\right)\right)
$$

and

$$
\left[\sigma_{L}\right]=c_{\text {top }}\left(\operatorname{Sym}^{l} U^{*}\right) \pi_{k *}\left(c_{t o p}\left(\pi_{k}^{*} \operatorname{Sym}^{d} U^{*} /\left(\pi_{k}^{*} \operatorname{Sym}^{l} U^{*} \otimes T_{k}\right)\right) c_{t o p}\left(\pi_{k}^{*} \operatorname{Sym}^{k} U^{*} / T_{k}\right)\right) .
$$

By using the setup in Section 1, the above facts can now be applied to the refined intersection product defined there. Notice that the following statement is purely pointwise on fixed $X_{0}$ and no deformation is involved.

Summary/Theorem 4.1'. Let $R_{X_{0}}$ be the refined intersection product in $A_{m}\left(F_{X_{0}}\right)$ as defined in Section 1. The contributions (equivalences) of $F_{K}$ and $F_{L}$ to $R_{X_{0}}$ are

$$
R_{X_{0}}^{F_{K}}=c_{t o p}\left(\operatorname{Sym}^{k} U^{*}\right) \pi_{l *}\left(c_{t o p}\left(\pi_{l}^{*} \operatorname{Sym}^{d} U^{*} /\left(\pi_{l}^{*} \operatorname{Sym}^{k} U^{*} \otimes T_{l}\right)\right) c_{t o p}\left(\pi_{l}^{*} \operatorname{Sym}^{l} U^{*} / T_{l}\right)\right)
$$

and

$$
R_{X_{0}}^{F_{L}}=c_{t o p}\left(\operatorname{Sym}^{l} U^{*}\right) \pi_{k *}\left(c_{t o p}\left(\pi_{k}^{*} \operatorname{Sym}^{d} U^{*} /\left(\pi_{k}^{*} \operatorname{Sym}^{l} U^{*} \otimes T_{k}\right)\right) c_{t o p}\left(\pi_{k}^{*} \operatorname{Sym}^{k} U^{*} / T_{k}\right)\right) \text {, }
$$

respectively. In particular, we have the canonical decomposition of $R_{X_{0}}$ as follows $(4.2)$

$$
\begin{aligned}
R_{X_{0}}= & c_{\text {top }}\left(\operatorname{Sym}^{k} U^{*}\right) \pi_{l *}\left(c_{t o p}\left(\pi_{l}^{*} \operatorname{Sym}^{d} U^{*} /\left(\pi_{l}^{*} \operatorname{Sym}^{k} U^{*} \otimes T_{l}\right)\right) c_{t o p}\left(\pi_{l}^{*} \operatorname{Sym}^{l} U^{*} / T_{l}\right)\right) \\
& +c_{t o p}\left(\operatorname{Sym}^{l} U^{*}\right) \pi_{k *}\left(c_{t o p}\left(\pi_{k}^{*} \operatorname{Sym}^{d} U^{*} /\left(\pi_{k}^{*} \operatorname{Sym}^{l} U^{*} \otimes T_{k}\right)\right) c_{t o p}\left(\pi_{k}^{*} \operatorname{Sym}^{k} U^{*} / T_{k}\right)\right) .
\end{aligned}
$$

Proof. Except in the case of $r=1$, it may be possible that $R_{X_{0}}$ will have $F_{K} \cap F_{L}$ as a distinguished variety. However, from the definitions of $\sigma_{K}$ and $\sigma_{L}$, we see that the subscheme of limiting $\mathbb{P}^{r}$ 's contained in $K \cap L$ must have a dimension strictly less than $m$. Therefore, the equivalence of $F_{K} \cap F_{L}$ would be zero in any case. This implies that the sum of $\left[\sigma_{K}\right]$ and $\left[\sigma_{L}\right]$ is indeed a decomposition of $R_{X_{0}}$. 
Question. There is one more decomposition of $R_{X_{0}}$ that is worth mentioning. If $F_{K}$ was a connected component of $F_{X_{0}}$, then the contribution of $F_{K}$ to $R_{X_{0}}$ would be

$$
R_{X_{0}}^{F_{K}}=\left\{c\left(\operatorname{Sym}^{d} U^{*}\right) \cup s\left(F_{K}, G\right)\right\}_{m} .
$$

This class is very easy to compute. In general, we can still decompose $R_{X_{0}}$ as the sum of the class above and the so-called residual intersection class $\mathbb{R}$. This is the celebrated residual intersection formula of Fulton-Laksov [F, Chapter 9] [FL] [L]. We can also compute the similar residual decomposition of $R_{X_{0}}$ for $F_{L}$. What are geometric meanings for such decompositions? We have computed some examples but could not give satisfactory answers.

\section{IDENTITIES IN CHARACTERISTIC CLASSES AND EXAMPLES}

In this section, we will derive some identities in characteristic classes from the results in previous sections and then compute a few examples with our formulas. From the splitting principle, those identities can be translated into identities in symmetric polynomials of $r+1$ variables and hence should hold for any vector bundle of rank $r+1$ over a manifold. It will be interesting to see if the identities can be verified by a more direct approach.

Since the image of $R_{X_{0}}$ in the Chow ring of $G(r+1, n+1)$ is always equal to the top Chern class of $\mathrm{Sym}^{d} U^{*}$, the results in the last section immediately imply the following family of identities.

Corollary 5.1. For any $d, k$, and $l$ with $d=k+l$, the following identity holds on the Chow ring of $G(r+1, n+1)$.

$$
\begin{aligned}
& c_{\text {top }}\left(\operatorname{Sym}^{d} U^{*}\right) \\
= & c_{\text {top }}\left(\operatorname{Sym}^{k} U^{*}\right) \pi_{l *}\left(c_{t o p}\left(\pi_{l}^{*} \operatorname{Sym}^{d} U^{*} /\left(\pi_{l}^{*} \operatorname{Sym}^{k} U^{*} \otimes T_{l}\right)\right) c_{t o p}\left(\pi_{l}^{*} \operatorname{Sym}^{l} U^{*} / T_{l}\right)\right) \\
+ & c_{\text {top }}\left(\operatorname{Sym}^{l} U^{*}\right) \pi_{k *}\left(c_{\text {top }}\left(\pi_{k}^{*} \operatorname{Sym}^{d} U^{*} /\left(\pi_{k}^{*} \operatorname{Sym}^{l} U^{*} \otimes T_{k}\right)\right) c_{t o p}\left(\pi_{k}^{*} \operatorname{Sym}^{k} U^{*} / T_{k}\right)\right) .
\end{aligned}
$$

Before giving examples, we will first rewrite formula (3.2) and formula (3.3) more explicitly in terms of elements in the Chow ring of $G(r+1, n+1)$. One of the reasons for doing this is because it will be useful in actual computations. Since the Chow ring of the projective bundle $\mathbb{P}\left(\operatorname{Sym}^{l} U^{*}\right)$ is bigger and more complicated than the Chow ring of the underlying space $G(r+1, n+1)$, in many cases, a direct computation on $G(r+1, n+1)$ will be faster than computing first on $\mathbb{P}\left(\operatorname{Sym}^{l} U^{*}\right)$ and then pushing it down. More importantly, by computing on $G(r+1, n+1)$ only, we eliminate the need to create the projective bundle $\mathbb{P}\left(\mathrm{Sym}^{l} U^{*}\right)$, which may take too much time and space on a computer, especially if the rank of $\mathrm{Sym}^{l} U^{*}$ is big.

For a given vector bundle $E$, we will use $c_{i}(E)$ to denote the $i^{\text {th }}$ Chern class of $E$ and $s_{i}(E)$ to denote the $i^{\text {th }}$ Segre class of $E$. Recall also that we identify an element in $A^{*}(G(r+1, n+1))$ with its dual in $A_{*}(G(r+1, n+1))$.

Proposition 5.3. Formula (3.2) can be rewritten directly in terms of elements in the Chow ring of $G(r+1, n+1)$ as

$$
\begin{aligned}
{\left[\sigma_{K}\right]=} & c_{r_{k}}\left(\operatorname{Sym}^{k} U^{*}\right) \sum_{i=0}^{r_{d}-r_{k}} \sum_{j=0}^{r_{l}-1} \sum_{h=0}^{r_{d}-r_{k}-i-j}\left(\begin{array}{c}
r_{d}-1-i \\
r_{k}-1+h
\end{array}\right) c_{i}\left(\operatorname{Sym}^{d} U^{*}\right) \\
& \times c_{j}\left(\operatorname{Sym}^{l} U^{*}\right) s_{h}\left(\operatorname{Sym}^{k} U^{*}\right) s_{r_{d}-r_{k}-h-i-j}\left(\operatorname{Sym}^{l} U^{*}\right),
\end{aligned}
$$


where

$$
\left\{\begin{array}{c}
r_{d}=\operatorname{rank}\left(\operatorname{Sym}^{d} U^{*}\right)=\left(\begin{array}{c}
r+d \\
r
\end{array}\right), \\
r_{k}=\operatorname{rank}\left(\operatorname{Sym}^{k} U^{*}\right)=\left(\begin{array}{c}
r+k \\
r
\end{array}\right), \\
r_{l}=\operatorname{rank}\left(\operatorname{Sym}^{l} U^{*}\right)=\left(\begin{array}{c}
r+l \\
r
\end{array}\right) .
\end{array}\right.
$$

The corresponding formula holds for $\left[\sigma_{L}\right]$ if we interchange $k$ and $l$.

Remark. Since $s_{i}(E)=0$ for all $i<0$, we may replace the range of $j$ by

$$
0 \leq j \leq \min \left\{r_{l}-1, r_{d}-r_{k}-i\right\}
$$

Proof. This is a straightforward computation with standard calculations on the Chern classes and the Segre classes. All the formulas used here can be found in Fulton's book [F]. From formula (3.2),

$$
\begin{aligned}
{\left[\sigma_{K}\right]=} & c_{\text {top }}\left(\operatorname{Sym}^{k} U^{*}\right) \pi_{l *}\left(c_{t o p}\left(\pi_{l}^{*} \operatorname{Sym}^{d} U^{*} /\left(\pi_{l}^{*} \operatorname{Sym}^{k} U^{*} \otimes T_{l}\right)\right) c_{t o p}\left(\pi_{l}^{*} \operatorname{Sym}^{l} U^{*} / T_{l}\right)\right) \\
= & c_{r_{k}}\left(\operatorname{Sym}^{k} U^{*}\right) \pi_{l *}\left(\sum_{i=0}^{r_{d}-r_{k}} c_{i}\left(\pi_{l}^{*} \operatorname{Sym}^{d} U^{*}\right) s_{r_{d}-r_{k}-i}\left(\pi_{l}^{*} \operatorname{Sym}^{k} U^{*} \otimes T_{l}\right)\right. \\
& \left.\times \sum_{j=0}^{r_{l}-1} c_{j}\left(\pi_{l}^{*} \operatorname{Sym}^{l} U^{*}\right) s_{r_{l}-1-j}\left(T_{l}\right)\right) \\
= & c_{r_{k}}\left(\operatorname{Sym}^{k} U^{*}\right) \pi_{l *}\left(\sum_{i=0}^{r_{d}-r_{k}} c_{i}\left(\pi_{l}^{*} \operatorname{Sym}^{d} U^{*}\right) \sum_{h=0}^{r_{d}-r_{k}-i}\left(\begin{array}{c}
r_{k}-1+r_{d}-r_{k}-i \\
r_{k}-1+h
\end{array}\right)\right. \\
& \left.\times s_{h}\left(\pi_{l}^{*} \operatorname{Sym}^{k} U^{*}\right) c_{1}\left(-T_{l}\right)^{r_{d}-r_{k}-i-h} \sum_{j=0}^{r_{l}-1} c_{j}\left(\pi_{l}^{*} \operatorname{Sym}^{l} U^{*}\right) c_{1}\left(-T_{l}\right)^{r_{l}-1-j}\right) \\
= & c_{r_{k}}\left(\operatorname{Sym}^{k} U^{*}\right) \sum_{i=0}^{r_{d}-r_{k}} \sum_{j=0}^{r_{l}-1} \sum_{h=0}^{r_{d}-r_{k}-i}\left(\begin{array}{c}
r_{d}-1-i \\
r_{k}-1+h
\end{array}\right) c_{i}\left(\mathrm{Sym}^{d} U^{*}\right) \\
& \times c_{j}\left(\mathrm{Sym}^{l} U^{*}\right) s_{h}\left(\mathrm{Sym}^{k} U^{*}\right) \pi_{l *}\left(c_{1}(\mathcal{O}(1))^{r_{d}-r_{k}+r_{l}-1-i-j-h}\right) \\
= & c_{r_{k}}\left(\operatorname{Sym}^{k} U^{*}\right) \sum_{i=0}^{r_{d}-r_{k}} \sum_{j=0}^{r_{l}-1} \sum_{h=0}^{r_{d}-r_{k}-i-j}\left(\begin{array}{c}
r_{d}-1-i \\
r_{k}-1+h
\end{array}\right) c_{i}\left(\mathrm{Sym}^{d} U^{*}\right) \\
& \times c_{j}\left(\operatorname{Sym}^{l} U^{*}\right) s_{h}\left(\operatorname{Sym}^{k} U^{*}\right) s_{r_{d}-r_{k}-i-j-h}\left(\mathrm{Sym}^{l} U^{*}\right) .
\end{aligned}
$$

In the case of $r=1$, we have

$$
r_{d}=d+1, \quad r_{k}=k+1, \quad r_{l}=l+1
$$


Corollary 5.5. For the case of lines,

$$
\begin{aligned}
{\left[\sigma_{K}\right]=} & c_{k+1}\left(\operatorname{Sym}^{k} U^{*}\right) \sum_{i=0}^{l} \sum_{j=0}^{l-i} \sum_{h=0}^{l-i-j}\left(\begin{array}{c}
d-i \\
k+h
\end{array}\right) c_{i}\left(\operatorname{Sym}^{d} U^{*}\right) c_{j}\left(\operatorname{Sym}^{l} U^{*}\right) \\
& \times s_{h}\left(\operatorname{Sym}^{k} U^{*}\right) s_{l-h-i-j}\left(\operatorname{Sym}^{l} U^{*}\right) .
\end{aligned}
$$

The corresponding formula holds for $\left[\sigma_{L}\right]$ if we interchange $k$ and $l$.

Corollary 5.1 can now be restated as well.

Corollary 5.7. For any set of non-negative integers $r, n, d, k$, and $l$ with $r<n$ and $d=k+l$, the following identity holds on the Chow ring of $G(r+1, n+1)$.

$$
\begin{aligned}
c_{\text {top }}\left(\operatorname{Sym}^{d} U^{*}\right)= & c_{r_{k}}\left(\operatorname{Sym}^{k} U^{*}\right) \sum_{i=0}^{r_{d}-r_{k}} \sum_{j=0}^{r_{l}-1} \sum_{h=0}^{r_{d}-r_{k}-i-j}\left(\begin{array}{c}
r_{d}-1-i \\
r_{k}-1+h
\end{array}\right) c_{i}\left(\operatorname{Sym}^{d} U^{*}\right) \\
& \times c_{j}\left(\operatorname{Sym}^{l} U^{*}\right) s_{h}\left(\operatorname{Sym}^{k} U^{*}\right) \\
& +c_{r_{l}}\left(\operatorname{Sym}^{l} U^{*}\right) \sum_{i=0}^{r_{d}-r_{k}-h-i-j} \sum_{j=0}^{r_{k}-1} \sum_{h=0}^{r_{d}-\operatorname{Sym}_{l}-i-j}\left(\begin{array}{c}
r_{d}-1-i \\
r_{l}-1+h
\end{array}\right) c_{i}\left(\operatorname{Sym}^{d} U^{*}\right) \\
& \times c_{j}\left(\operatorname{Sym}^{k} U^{*}\right) s_{h}\left(\operatorname{Sym}^{l} U^{*}\right) s_{r_{d}-r_{l}-h-i-j}\left(\operatorname{Sym}^{k} U^{*}\right),
\end{aligned}
$$

where

$$
\left\{\begin{array}{c}
r_{d}=\operatorname{rank}\left(\operatorname{Sym}^{d} U^{*}\right)=\left(\begin{array}{c}
r+d \\
r
\end{array}\right), \\
r_{k}=\operatorname{rank}\left(\operatorname{Sym}^{k} U^{*}\right)=\left(\begin{array}{c}
r+k \\
r
\end{array}\right), \\
r_{l}=\operatorname{rank}\left(\operatorname{Sym}^{l} U^{*}\right)=\left(\begin{array}{c}
r+l \\
r
\end{array}\right) .
\end{array}\right.
$$

Moreover, this gives the canonical decomposition of $R_{X_{0}}$.

Remark. We think identities (5.2) and (5.8) should hold for any vector bundle of rank $r+1$ over a general manifold. In fact, no relations in Chow rings should be needed and they should hold as identities of symmetric polynomials as we have seen in [W] for the case of $r=1$. However, we do not know whether such identities would have further significant meanings in general. It will be very interesting if one can find other applications of such identities in different settings.

We will now compute some examples using either Theorem 3.1 or Proposition 5.3. Most of our computations are done by using the schubert package [KS] on Maple. It is very easy to write a schubert code based on formula (3.2) and formula (3.3). On the other hand, although formulas given in Proposition 5.3 are more complicated, their computations in many cases are much faster. To keep track of the degrees of the hypersurfaces in our degeneration, we will use $R(d, k)$ for $\left[\sigma_{K}\right]$ and $R(d, l)$ for $\left[\sigma_{L}\right]$ in the following examples. In theory, one can compute any $R(d, k)$ and $R(d, l)$ in $A^{*}(G(r+1, n+1))$. In practice, however, one will find out quickly that $d$ or $r$ cannot be set too large even with the help of powerful computers. 
Example 1. Degenerations of hypersurfaces and their lines $(r=1)$.

In this case, we got the same results as given in $[\mathrm{W}]$ and one can find detailed examples, such as degenerations of cubic surfaces, or quintic threefolds, there. Since the formulas used here are not the same as given in [W], it is a reassurance to see that we do get the same results. Comparing Corollary 5.5 of this paper and Theorem 1.1 in $[\mathrm{W}]$, we see that the formulas here are more complicated and have many extra terms. Those extra terms must actually cancel out each other. However, it does not appear to be trivial to prove directly that the formulas given in Corollary 5.5 are actually equal to the corresponding formulas in [W]. Once again, we think that they should be equal to each other as polynomials and hence the equations should hold for general vector bundles of rank 2 .

Example 2. Degenerations of a generic quartic hypersurface in $\mathbb{P}^{7}$ and its $\mathbb{P}^{2}$ 's.

See also [K3] for a computation by geometric methods. It is that computation which leads to the discovery of our formulas.

There are $3,297,280 \mathbb{P}^{2}$ 's on a generic quartic hypersurface in $\mathbb{P}^{7}$. This is given by the degree of $c_{t o p}\left(\operatorname{Sym}^{4} U^{*}\right)$ on $A^{*}(G(3,8))$. There are two degenerations and computations using our representations show that

Case $1, K$ is a cubic and $L$ is a plane: $\quad R(4,3)=483,840, R(4,1)=$ $2,813,440$.

Case $2, K$ and $L$ are quadrics: $\quad R(4,2)=1,648,640$.

Notice that in each of the cases above we do have that

$$
R(4, k)+R(4, l)=3,297,280=c_{t o p}\left(\operatorname{Sym}^{7} U^{*}\right)
$$

as expected.

Example 3. Degenerations of a generic quadric in $\mathbb{P}^{n}, n \geq 3$, and its $\mathbb{P}^{2}$ 's

To eliminate doubtful feelings one might have towards computers, this example has been double checked by hand and details are given below.

Let

$$
c_{1}\left(U^{*}\right)=x, \quad c_{2}\left(U^{*}\right)=y, \quad c_{3}\left(U^{*}\right)=z .
$$

It is easy to compute that

$$
\left\{\begin{aligned}
c_{1}\left(\operatorname{Sym}^{2} U^{*}\right) & =4 x, \\
c_{2}\left(\operatorname{Sym}^{2} U^{*}\right) & =5\left(x^{2}+y\right), \\
c_{3}\left(\operatorname{Sym}^{2} U^{*}\right) & =2 x^{3}+11 x y+7 z, \\
\vdots & \vdots \\
c_{6}\left(\operatorname{Sym}^{2} U^{*}\right) & =8 z(x y-z) .
\end{aligned}\right.
$$

Therefore, the class of $\mathbb{P}^{2}$ 's on a generic quadric is equal to

$$
8 x y z-8 z^{2} .
$$

On the other hand, to compute the class of limiting $\mathbb{P}^{2}$ 's when the quadric degenerates into two $(n-1)$-planes $K$ and $L$, we set

$$
r=d=2, \quad l=k=1
$$


in (5.4) of Proposition 5.3. This gives us

$$
R(2,1)=c_{3}\left(U^{*}\right) \sum_{i=0}^{3} \sum_{j=0}^{2} \sum_{h=0}^{3-i-j}\left(\begin{array}{c}
5-i \\
2+h
\end{array}\right) c_{i}\left(\operatorname{Sym}^{2} U^{*}\right) c_{j}\left(U^{*}\right) s_{h}\left(U^{*}\right) s_{3-h-i-j}\left(U^{*}\right) .
$$

It is easy to see that

$$
\left\{\begin{array}{l}
s_{1}\left(\mathrm{Sym}^{2} U^{*}\right)=-x, \\
s_{2}\left(\mathrm{Sym}^{2} U^{*}\right)=x^{2}-y, \\
s_{3}\left(\mathrm{Sym}^{2} U^{*}\right)=-x^{3}+2 x y-z,
\end{array}\right.
$$

Substituting (5.9) and (5.11) into (5.10) and simplifying, we finally have

$$
R(2,1)=4 x y z-4 z^{2}
$$

as expected.

We have purposefully done our computations without using the Schubert calculus. Therefore, this also verifies the generalization of identity (5.8) to any vector bundle of rank 3 in the case of $d=2$.

Remark. It is well-known that there is no $\mathbb{P}^{2}$ in a generic quadric in $\mathbb{P}^{n}$ if $n<5$. This can be seen by using the Schubert calculus. On $A_{*}(G(3, n+1))$,

$$
c_{6}\left(\operatorname{Sym}^{2} U^{*}\right)=8 z(x y-z)=8 \sigma_{3,2,1} .
$$

(In fact, $8 \sigma_{3,2,1}$ is equal to the class of $\mathbb{P}^{2}$ 's in any smooth quadric. See $[\mathrm{GH}$, Chapter 6] for computations using geometric methods.) Hence, this class is zero if $n<5$.

Our last example shows something interesting.

Example 4. Degenerations of a generic cubic hypersurface in $\mathbb{P}^{8}$ and its $\mathbb{P}^{3}$ 's.

By computing the degree of $c_{t o p}\left(\operatorname{Sym}^{3} U^{*}\right)$ on $A^{*}(G(4,9))$, we see that there are $321,489 \mathbb{P}^{3}$ 's on a generic cubic hypersurface in $\mathbb{P}^{8}$. When the cubic degenerates into the union of a quadric and a plane, computations from our formulas yield that

$$
R(3,2)=0, \quad R(3,1)=321,489 .
$$

The result is a surprise since it says that all limiting $\mathbb{P}^{3}$ 's are in the plane and none of them is in the quadric. In terms of intersection theory, that means the equivalence of $F_{K}$ for $R_{X_{0}}$ is zero. Notice that $\mathrm{Sym}^{d} U^{*}$ is a bundle generated by sections so all equivalences must be non-negative classes. Our example gives the extreme case and implies that $\operatorname{Sym}^{d} U^{*}$ (and hence $U^{*}$ ) is not a positive bundle.

\section{Appendix: Schubert CODE}

In this appendix, we will give schubert code for Example 3 and Example 4 of Section 5. Formula (4.4) of Proposition 5.3 will be applied for Example 3 and Formula (3.2) and formula (3.3) of Theorem 3.1 will be used for Example 4. By 
changing corresponding parameters $r, n, d, k$, and $l$, those programs can be converted easily to compute other examples. Schubert or Maple commands that a user is required to type during a Maple session will be indicated by the line beginning with the Maple prompt ">". Comments will be indicated by the line beginning with "\#". In many places, we have used the command "latex" to make tex-format output. One should leave them out if regular output is desired. We want to thank Katz and Strømme for making this wonderful Maple package available. For those who may be interested in getting the current version of schubert, we want to point out that it is available by using ftp to ftp.math.okstate.edu with user=anonymous, password $=<$ your email address $>$, cd pub/schubert, then get the files there. One can find instructions on how to install the package from the manual.

A note of caution. Schubert uses notation from the school of Grothendieck. Since notation in this paper primarily follows from Fulton's book, we have to use the dual to covert between them in many places. This may cause some confusion. It could easily lead to wrong answers if one is not careful about this.

Schubert code for Example 3. Degenerations of a generic quadric in $\mathbb{P}^{n}, n=4$, and its $\mathbb{P}^{2}$ 's. Results will be same for all $n>4$ except for the last computation.

\# Construct $G(3,5)=G c$ with $U^{*}=Q c$ and $c_{i}\left(U^{*}\right)=c_{i}$.

$>\operatorname{latex}(\operatorname{grass}(3,5, \mathrm{c}, \mathrm{mon}))$;

currentvariety_is Gc, DIM is 6

\# Construct $\mathrm{Sym}^{2} U^{*}=s 2$.

$>\mathrm{s} 2:=\operatorname{symm}(2, \mathrm{Qc})$ :

\# The class of $\mathbb{P}^{2}$ 's in a generic quadric is equal to the top chern class of $\operatorname{Sym}^{2} U^{*}$.

$>$ cl_P2_in_X2:=chern $(\operatorname{rank}(\mathrm{s} 2), \mathrm{s} 2)$ :

$>\operatorname{latex}\left({ }^{\prime \prime}\right)$;

$$
c l \_P 2 \_i n \_X 2=8 c_{3} c_{1} c_{2}-8 c_{3}^{2}
$$

\# Use formula (5.10) to compute the class of limiting $\mathbb{P}^{2}$ 's in a hyperplane which should

\# be equal to one half of the class above.

$>\mathrm{f}:=0$ :

$>$ for i from 0 to 3 do

$>$ for $\mathrm{j}$ from 0 to $\min (2,3-\mathrm{i})$ do

$>$ for $\mathrm{h}$ from 0 to $3-\mathrm{i}-\mathrm{j}$ do

$>\mathrm{f}:=\mathrm{f}+$

$>\operatorname{binomial}(5-\mathrm{i}, 2+\mathrm{h}) * \operatorname{chern}(\mathrm{i}, \mathrm{s} 2) * \operatorname{chern}(\mathrm{j}, \mathrm{Qc}) * \operatorname{segre}(\mathrm{h}, \operatorname{dual}(\mathrm{Qc})) * \operatorname{segre}(3-\mathrm{h}-\mathrm{i}-\mathrm{j}, \operatorname{dual}(\mathrm{Qc}))$

$>$ od od od:

$>$ cl_limiting_P2_in_X1:=expand $(\operatorname{chern}(3, \mathrm{Qc}) * \mathrm{f})$ :

$>\operatorname{latex}\left({ }^{\prime \prime}\right)$;

$$
\text { cl_limiting_P2_in_X1 }=4 c_{3} c_{1} c_{2}-4 c_{3}{ }^{2}
$$

\# In our case of $n=4, \operatorname{dim}(G(3,5))=\operatorname{rank}\left(\operatorname{Sym}^{2} U^{*}\right)$, so the class above gives a number.

$>$ number:=integral(Gc,cl_limiting_P2_in_X1):

$>\operatorname{latex}\left({ }^{\prime \prime}\right)$; 


$$
\text { number }=0
$$

$>$ quit;

Schubert code for Example 4. Degenerations of a generic cubic hypersurface in $\mathbb{P}^{8}$ and its $\mathbb{P}^{3}$ 's.

\# Construct $G(4,9)=G c$ with $U^{*}=Q c$ and $c_{i}\left(U^{*}\right)=c_{i}$.

$>\operatorname{latex}(\operatorname{grass}(4,9, \mathrm{c}, \mathrm{mon}))$;

currentvariety_is Gc, DIM is 20

\# Construct $\mathrm{Sym}^{3} U^{*}=s 3$.

$>\mathrm{s} 3:=\operatorname{symm}(3, \mathrm{Qc})$ :

\# The class of $\mathbb{P}^{3}$ 's in a generic cubic hypersurface is equal to the top chern class \# of $\mathrm{Sym}^{3} U^{*}$.

$>$ cl_P3_in_X3:=chern $(20, \mathrm{~s} 3)$ :

$>\operatorname{latex}\left({ }^{\prime \prime}\right)$;

$$
\begin{aligned}
c l_{-} P 3 \_i n \_X 3= & -1296 c_{4}{ }^{2} c_{3}{ }^{3} c_{1}{ }^{3}-2592 c_{4} c_{3}{ }^{3} c_{1} c_{2}{ }^{3}+17496 c_{4}{ }^{2} c_{3}{ }^{4} \\
& +1296 c_{4}{ }^{3} c_{2}{ }^{4}+15552 c_{4}{ }^{2} c_{3}{ }^{2} c_{2} c_{1}{ }^{4}+2592 c_{4} c_{3}{ }^{2} c_{2}{ }^{4} c_{1}{ }^{2} \\
& +50625 c_{4}{ }^{5}+2592 c_{4}{ }^{2} c_{3}{ }^{2} c_{2}{ }^{3}-14580 c_{4}{ }^{3} c_{3}{ }^{2} c_{2} \\
& +36045 c_{4}{ }^{4} c_{2} c_{1}{ }^{2}+17496 c_{4}{ }^{3} c_{2} c_{1}{ }^{6}+34425 c_{4}{ }^{4} c_{1} c_{3} \\
& +1296 c_{4}{ }^{2} c_{2}{ }^{5} c_{1}{ }^{2}-17496 c_{4}{ }^{3} c_{3} c_{1}{ }^{5}-81162 c_{4}{ }^{3} c_{3}{ }^{2} c_{1}{ }^{2} \\
& -13608 c_{4}{ }^{3} c_{2}{ }^{3} c_{1}{ }^{2}+2592 c_{4}{ }^{2} c_{2}{ }^{4} c_{1}{ }^{4}-14580 c_{4}{ }^{3} c_{2}{ }^{2} c_{1}{ }^{4} \\
& -17496 c_{4} c_{1} c_{3}{ }^{5}-2592 c_{4} c_{3}{ }^{4} c_{1}{ }^{4}+87966 c_{4}{ }^{3} c_{3} c_{2} c_{1}{ }^{3} \\
& +3888 c_{4}{ }^{3} c_{3} c_{1} c_{2}{ }^{2}-16200 c_{4}{ }^{4} c_{2}{ }^{2}+17496 c_{4}{ }^{4} c_{1}{ }^{4} \\
& +2916 c_{4}{ }^{2} c_{3}{ }^{2} c_{2}{ }^{2} c_{1}{ }^{2}-1296 c_{4}{ }^{2} c_{3} c_{1} c_{2}{ }^{4}-11664 c_{4}{ }^{2} c_{3} c_{2}{ }^{2} c_{1}{ }^{5} \\
& +2916 c_{4}{ }^{2} c_{3}{ }^{3} c_{1} c_{2}-14904 c_{4}{ }^{2} c_{3} c_{2}{ }^{3} c_{1}{ }^{3}+5184 c_{4} c_{3}{ }^{2} c_{2}{ }^{3} c_{1}{ }^{4} \\
& +29160 c_{4} c_{3}{ }^{4} c_{2} c_{1}{ }^{2}+2592 c_{4} c_{3}{ }^{3} c_{2} c_{1}{ }^{5}-16848 c_{4} c_{3}{ }^{3} c_{2}{ }^{2} c_{1}{ }^{3}
\end{aligned}
$$
in

\# Since $\operatorname{dim}(G(4,9))=\operatorname{rank}\left(\operatorname{Sym}^{3} U^{*}\right)$, the class above gives the number of $\mathbb{P}^{3}$ 's

\# a generic cubic hypersurface in $\mathbb{P}^{8}$.

$>$ total_number:=integral(Gc,cl_P3_in_X3):

$>\operatorname{latex}\left({ }^{\prime \prime}\right)$;

$$
\text { total_number }=321489
$$

\# Construct $\mathrm{Sym}^{2} U^{*}=s 2$.

$>\mathrm{s} 2:=\operatorname{symm}(2, \mathrm{Qc})$ :

\# Construct the projective bundle $\mathbb{P}\left(U^{*}\right)$ with the projection map $\pi_{1}=P 1$ and the

\# tautological bundle $T_{1}=z 1$.

$>\operatorname{Proj}(\mathrm{P} 1, \operatorname{dual}(\mathrm{Qc}), \mathrm{z} 1)$ :

\# Use formula (3.2) to compute the class and the number of limiting $\mathbb{P}^{3}$ 's in a generic 
\# quadric.

$>$ cl_limiting_P3_in_X2:=

$>\operatorname{expand}(\operatorname{chern}(10, \mathrm{~s} 2) *$ lowerstar $(\mathrm{P} 1, \operatorname{chern}(10, \mathrm{~s} 3-\mathrm{s} 2 \& * \mathrm{o}(-\mathrm{z} 1)) * \operatorname{chern}(3, \mathrm{Qc}-\mathrm{o}(-\mathrm{z} 1))))$ :

$>\operatorname{latex}\left({ }^{\prime \prime}\right)$

$$
\begin{aligned}
\text { cl_limiting_P3_in_X2 }= & -12528 c_{4}{ }^{2} c_{3}{ }^{3} c_{1}{ }^{3}-1728 c_{4} c_{3}{ }^{3} c_{1} c_{2}{ }^{3}+11664 c_{4}{ }^{2} c_{3}{ }^{4} \\
& +17280 c_{4}{ }^{2} c_{3}{ }^{2} c_{2} c_{1}{ }^{4}+1728 c_{4} c_{3}{ }^{2} c_{2}{ }^{4} c_{1}{ }^{2}+1728 c_{4}{ }^{2} c_{3}{ }^{2} c_{2}{ }^{3} \\
& -10800 c_{4}{ }^{3} c_{3}{ }^{2} c_{2}-10800 c_{4}{ }^{4} c_{2} c_{1}{ }^{2}+8640 c_{4}{ }^{3} c_{2} c_{1}{ }^{6} \\
& -864 c_{4}{ }^{3} c_{3} c_{1}{ }^{5}-12960 c_{4}{ }^{3} c_{3}{ }^{2} c_{1}{ }^{2}+1728 c_{4}{ }^{3} c_{2}{ }^{3} c_{1}{ }^{2} \\
& -1728 c_{4}{ }^{2} c_{3}{ }^{2} c_{1}{ }^{6}+7776 c_{4}{ }^{3} c_{2}{ }^{2} c_{1}{ }^{4}-11664 c_{4} c_{1} c_{3}{ }^{5} \\
& -1728 c_{4} c_{3}{ }^{4} c_{1}{ }^{4}+32400 c_{4}{ }^{3} c_{3} c_{2} c_{1}{ }^{3}+10800 c_{4}{ }^{3} c_{3} c_{1} c_{2}{ }^{2} \\
& -24624 c_{4}{ }^{4} c_{1}{ }^{4}-1728 c_{4}{ }^{2} c_{3} c_{1} c_{2}{ }^{4}-12096 c_{4}{ }^{2} c_{3} c_{2}{ }^{2} c_{1}{ }^{5} \\
& -3888 c_{4}{ }^{2} c_{3}{ }^{3} c_{1} c_{2}-9504 c_{4}{ }^{2} c_{3} c_{2}{ }^{3} c_{1}{ }^{3}+3456 c_{4} c_{3}{ }^{2} c_{2}{ }^{3} c_{1}{ }^{4} \\
& +19440 c_{4} c_{3}{ }^{4} c_{2} c_{1}{ }^{2}+1728 c_{4} c_{3}{ }^{3} c_{2} c_{1}{ }^{5}-11232 c_{4} c_{3}{ }^{3} c_{2}{ }^{2} c_{1}{ }^{3}
\end{aligned}
$$

$>$ number_X2:=integral(Gc,cl_limiting_P3_in_X2):

$>\operatorname{latex}\left({ }^{\prime \prime}\right)$;

$$
\text { number_X2 = } 0
$$

\# Construct the projective bundle $\mathbb{P}\left(\operatorname{Sym}^{2} U^{*}\right)$ with the projection map $\pi_{2}=P 2$ and the

\# tautological bundle $T_{2}=z 2$.

$>\operatorname{Proj}(\mathrm{P} 2, \operatorname{dual}(\mathrm{s} 2), \mathrm{z} 2)$ :

\# Use formula (3.3) to compute the class and the number of limiting $\mathbb{P}^{3}$ 's in a generic

\# hyperplane.

$>$ cl_limiting_P3_in_X1:=

$>\operatorname{expand}(\operatorname{chern}(4, \mathrm{Qc}) *$ lowerstar $(\mathrm{P} 2, \operatorname{chern}(16, \mathrm{~s} 3-\mathrm{Qc} \& * \mathrm{o}(-\mathrm{z} 2)) * \operatorname{chern}(9, \mathrm{~s} 2-\mathrm{o}(-\mathrm{z} 2))))$ :

$>\operatorname{latex}\left({ }^{\prime \prime}\right)$;

$$
\begin{aligned}
\text { cl_limiting_P3_in_X1 }= & 11232 c_{4}{ }^{2} c_{3}{ }^{3} c_{1}{ }^{3}-864 c_{4} c_{3}{ }^{3} c_{1} c_{2}{ }^{3}+5832 c_{4}{ }^{2} c_{3}{ }^{4} \\
& +1296 c_{4}{ }^{3} c_{2}{ }^{4}-1728 c_{4}{ }^{2} c_{3}{ }^{2} c_{2} c_{1}{ }^{4}+864 c_{4} c_{3}{ }^{2} c_{2}{ }^{4} c_{1}{ }^{2} \\
& +50625 c_{4}{ }^{5}+864 c_{4}{ }^{2} c_{3}{ }^{2} c_{2}{ }^{3}-3780 c_{4}{ }^{3} c_{3}{ }^{2} c_{2} \\
& +46845 c_{4}{ }^{4} c_{2} c_{1}{ }^{2}+8856 c_{4}{ }^{3} c_{2} c_{1}{ }^{6}+34425 c_{4}{ }^{4} c_{1} c_{3} \\
& +1296 c_{4}{ }^{2} c_{2}{ }^{5} c_{1}{ }^{2}-16632 c_{4}{ }^{3} c_{3} c_{1}{ }^{5}-68202 c_{4}{ }^{3} c_{3}{ }^{2} c_{1}{ }^{2} \\
& -15336 c_{4}{ }^{3} c_{2}{ }^{3} c_{1}{ }^{2}+1728 c_{4}{ }^{2} c_{3}{ }^{2} c_{1}{ }^{6}+2592 c_{4}{ }^{2} c_{2}{ }^{4} c_{1}{ }^{4} \\
& -22356 c_{4}{ }^{3} c_{2}{ }^{2} c_{1}{ }^{4}-5832 c_{4} c_{1} c_{3}{ }^{5}-864 c_{4} c_{3}{ }^{4} c_{1}{ }^{4} \\
& +55566 c_{4}{ }^{3} c_{3} c_{2} c_{1}{ }^{3}-6912 c_{4}{ }^{3} c_{3} c_{1} c_{2}{ }^{2}-16200 c_{4}{ }^{4} c_{2}{ }^{2} \\
& +42120 c_{4}{ }^{4} c_{1}{ }^{4}+2916 c_{4}{ }^{2} c_{3}{ }^{2} c_{2}{ }^{2} c_{1}{ }^{2}+432 c_{4}{ }^{2} c_{3} c_{1} c_{2}{ }^{4} \\
& +432 c_{4}{ }^{2} c_{3} c_{2}{ }^{2} c_{1}{ }^{5}+6804 c_{4}{ }^{2} c_{3}{ }^{3} c_{1} c_{2}-5400 c_{4}{ }^{2} c_{3} c_{2}{ }^{3} c_{1}{ }^{3} \\
& +1728 c_{4} c_{3}{ }^{2} c_{2}{ }^{3} c_{1}{ }^{4}+9720 c_{4} c_{3}{ }^{4} c_{2} c_{1}{ }^{2}+864 c_{4} c_{3}{ }^{3} c_{2} c_{1}{ }^{5} \\
& -5616 c_{4} c_{3}{ }^{3} c_{2}{ }^{2} c_{1}{ }^{3}
\end{aligned}
$$


$>$ number_X1:=integral(Gc,cl_limiting_P3_in_X1):

$>\operatorname{latex}\left({ }^{\prime \prime}\right)$

number_X1 $=321489$

$>$ quit;

\section{REFERENCES}

[COGP] P. Candelas, X. de la Ossa, P. Green, and L. Parkes, A pair of Calabi-Yau manifolds as an exactly soluble superconformal theory, Nuclear Phys. B 359 (1991), 21-74.

[F] W. Fulton, Intersection theory, Springer-Verlag, New York, 1984.

[FL] W. Fulton and D. Laksov, Residual intersections and the double point formula, in Real and Complex Singularities, Oslo 1976 (1977), P. Holm (ed.) Sijthoff and Noordhoff, 171178.

[FM] W. Fulton and R. MacPherson, Intersecting cycles on an algebraic variety, in Real and Complex Singularities, Oslo 1976 (1977), P. Holm (ed.) Sijthoff and Noordhoff, 179-197.

[GH] P. Griffiths and J. Harris, Principles of algebraic geometry, John Wiley and Sons, New York, 1978.

[GMP] B. Greene, D. Morrison, and M. Plesser, Mirror manifolds in higher dimension, preprint.

[K1] S. Katz, Degenerations of quintic threefolds and their lines, Duke Math. Jour. 50 (1983), 1127-1135.

[K2] S. Katz, Iteration of multiple point formulas and applications to conics, Algebraic Geometry, Sundance 1986, Lecture Notes in Math. 1311 (1988), 147-155.

[K3] S. Katz, Applications of schubert, Notes from RGI, Amherst, MA (1992).

[KS] S. Katz and S. Strømme, Schubert: a maple package for interesection theory. Available by anonymous ftp from ftp.math.okstate.edu, cd pub/schubert.

[L] D. Laksov, Residual intersections and Todd's formula for the double locus of a morphism, Acta. Math. 140 (1978), 75-92.

[La] R. Lazarsfeld, Excess intersection of divisors, Compositio Math. 43 (1981), 281-296.

[M1] D. Morrison, Mirror symmetry and rational curves on quintic threefolds: A guide for mathematicians, Duke preprint DUK-K-91-01 (July 1991).

[M2] D. Morrison, Picard-Fuchs equations and mirror maps for hypersurfaces, Duke preprint DUK-M-91-14 (October 1991).

[W] X. Wu, Chern classes and degenerations of hypersurfaces and their lines, Duke Math. Jour. 67 (1992), 633-652.

Department of Mathematics, University of South Carolina, Columbia, SC 29208

E-mail address: wux@milo.math.scarolina.edu 